\title{
Philosophiques
}

\section{Alfred Schmidt, Le concept de nature chez Marx, Paris, P.U.F. (coll. « Philosophie d'aujourd'hui »), 1994, 270 p.}

\section{Louis Desmeules}

Volume 24, numéro 1, printemps 1997

Avez-vous lu Rawls?

URI : https://id.erudit.org/iderudit/027436ar

DOI : https://doi.org/10.7202/027436ar

Aller au sommaire du numéro

Éditeur(s)

Société de philosophie du Québec

ISSN

0316-2923 (imprimé)

1492-1391 (numérique)

Découvrir la revue

Citer ce compte rendu

Desmeules, L. (1997). Compte rendu de [Alfred Schmidt, Le concept de nature chez Marx, Paris, P.U.F. (coll. « Philosophie d'aujourd'hui »), 1994, 270 p.]

Philosophiques, 24(1), 198-200. https://doi.org/10.7202/027436ar d'utilisation que vous pouvez consulter en ligne.

https://apropos.erudit.org/fr/usagers/politique-dutilisation/ 
Alfred Schmidt, Le concept de nature chez Marx, Paris, P.U.F. (coll. " Philosophie d'aujourd'hui "), 1994, 270 p.

Ce livre est une traduction d'une thèse de doctorat en philosophie écrite en 1957-1960 dans la lignée des travaux de l'école de Francfort (sous la direction d'Adomo et de Horkheimer) et publié pour la première fois en 1962. Ayant été l'objet de plusieurs révisions, la traduction actuelle se veut davantage fidèle au texte original. En plus de quatre grandes divisions, le livre comporte une préface à l'édition française dans laquelle l'auteur tente une mise à jour du matérialisme à la lumière des préoccupations écologiques actuelles. A été également ajouté un appendice (p. 221-257) pour faire suite à la controverse 
(Paris 1961), parue dans Marxisme et existentialisme (1962), entre Sartre, Garaudy, Hyppolite, Vigier et Orcel sur les relations entre l'histoire et la nature dans le matérialisme dialectique. Grosso modo, l'auteur s'accorde avec la position de Sartre et sa méthode " progressive-régressive ". Il y dénonce aussi les dangers du stalinisme. Le marxisme classique avait tendance à accorder à la croissance des forces productives "un rôle carrément métaphysique "(p. 2). L'auteur appuie sa thèse en mettant l'accent sur les cuvres tardives de Marx. en particulier le Capital et les Fondements de la critique de l'économie politique (1857-1859).

Le premier chapitre (p. 33-90) dégage le lien nié par beaucoup d'interprètes entre le matérialisme de Marx et le matérialisme philosophique en général. Marx cherchait à faire culminer les sciences de la nature et les sciences de l'homme en une seule science. Mais la vision du concept de nature chez Marx est éloignée de celle de Darwin ou de Hobbes, par exemple. "Pour Marx, la nature humaine ne connaît pas de données rigides, ni dans le domaine mental, ni dans le domaine matériel et biologique " (p. 71). Comme pour Hegel, l'histoire ne se déroule pas en ligne droite, mais dialectiquement. C'est pourquoi Marx voit le concept de nature relativement à l'activité humaine. Dans son élucidation du matérialisme philosophique, l'auteur s'applique à marquer le passage : HegelFeuerbach-Marx. Pour Hegel, la nature est le moment de l'extériorisation que l'idée parcourt comme abstraitement général pour revenir en soi dans l'esprit (p. 39). Avec Feuerbach, les formes logiques deviennent les fonctions d'êtres humains périssables, ce qui demeure abstrait pour Marx. La nature devient, pour Marx, un moment de la praxis humaine et en même temps la totalité de ce qui est " (p. 44).

La section B critique la dialectique de la nature chez Engels. L'auteur présente la position d'Engels dans le cadre d'une tentative pour donner une suite dialectique à la forme systématique du matérialisme français des Lumières (Holbach). Mais " la dialectique de la nature chez Engels reste une manière de voir les choses tout en leur restant extérieur * (p. 77). Engels interprète à partir du résultat des sciences, tandis que Marx, hégélien, critique de l'intérieur sans dériver des contenus de l'économie politique.

Le deuxième chapitre (p.91-130) êvalue les limites de la dialectique naturelle. Il s'agit de faire fonctionner la dialectique en montrant le travail qu'effectue Marx à partir de la réalité économique. La section $\mathrm{B}$ pose le difficile problème du statut de lontologie chez Marx qui demeurera en arrière-fond. Comment souligner d'abord le caractère non ontologique du matérialisme de Marx et parler ensuite d'ontologie, mème négative ? " La contradiction est dans les faits : elle ne peut être supprimée par la terminologie" (p. 22), nous répondait déjà l'auteur dans sa préface à la seconde édition allemande.

Dans le troisième chapitre (p. 131-171), l'auteur présente une série de considérations touchant la thérie de la connaissance chez Marx. Comment lier. la connaissance du processus naturel et celle du monde ? La conscience n'est pas figée mais elle se modifie historiquement. La section $\mathrm{C}$ tente ensuite de distinguer le mouvement des catégories de celui de la réalité qu'elles reproduisent. Le chapitre IV (p. 173-220) aborde la question de lutopie et l'on y reconnait qu'il y a chez Marx * une analyse lucide des conditions de la possibilité d'une liberté concrète $*(p .181)$. Cette section relève avec prudence certaines caractéristiques pour la création d'une sociēté future (travail et éducation), mais est surtout concentrée en une critique de la philosophie de Ernst Bloch.

Dans l'ensemble, Bloch est l'auteur le plus souvent citè après Hegel lau moins 22 fois). Rappelons que Bloch est un des théoriciens accusès, par Adorno 
et Hordheimer, d'improviser et d'être trop "romantique " (voir Rolf Wiggershaus, L'École de Francfort, histoire, développement, signification, Paris, P.U.F., 1993). Il fait donc partie des exclus de l'École de Francfort avec Benjamin, entre autres. Le problème de l'ontologie est posé dans l'optique d'une distanciation de Bloch. L'auteur reproche à Bloch sa compréhension trop "métaphysique " (p. 57). Bloch s'adonnerait à la croyance idéaliste qu'il y aurait " un fondement du monde, un principe de l'être s'engendrant lui-même " (p. 213). Bref, il reproche à Bloch de ne pas ètre clair, de s'éloigner de Marx (p. 213-217). Pourtant, il reconnait son importance dans le traitement de la question de l'utopie et la justesse de son raisonnement (p. 62).

En réalité, Bloch ne fait qu'assumer les conséquences de la pensée d'un monde sans domination ni hiérarchie. Le fondement de la philosophie blochienne est un fondement ouvert : "la catégorie de possibilité est fondation du fondement " (voir Experimentum mundi, d'Ernst Bloch), ce qui n'a rien à voir avec l'idéalisme critique de l'auteur. Il aurait fallu consulter. entre autres. les travaux de Gérard Raulet et d'Arno Munster pour avoir une présentation plus juste de la philosophie de Bloch. La question du vers quoi (du sens) reste ici en suspens sans le support d'une eschatologie conséquente. À cet égard, la fin est plutôt choquante (p. 216), car elle relativise le problème de l'homme et de la nature dans une praxis qui apparaît ainsi déconnectée.

Néanmoins, l'ouvrage montre qu'il n'y a pas de rupture absolue entre la pensée du jeune Marx et le Marx de la maturité. Il se présente comme une contribution à l'interprétation philosophique de Marx qui a le mérite de convoquer Marx autour des préoccupations écologiques actuelles : " Résurrection de la nature, humanisation de la nature, naturalisation de l'homme [...] De leur réussite dépend l'entrée de l'humanité dans un monde plus rationnel, et même la survie de l'humanité " (p. 24).

Louis Desmeules

Département de philosophie

Collège de Sherbrooke 